\title{
Proposed Use of Thickener According to Fluid Intake on Videofluoroscopic Swallowing Studies: Preliminary Study in Normal Healthy Persons
}

\author{
Kyung Duck Lee, MD', Sun Hong Song, MD, PhD ${ }^{1}$, Jung Hoi Koo, MD, PhD', \\ Hee Seon Park, $\mathrm{MD}^{1}$, Jae Sin Kim, $\mathrm{RN}^{1}$, Ki Hyo Jang, $\mathrm{PhD}^{2}$
}

${ }^{1}$ Department of Rehabilitation Medicine, Gangneung Asan Hospital, University of Ulsan College of Medicine, Gangneung;

${ }^{2}$ Department of Food \& Nutrition, College of Health \& Welfare, Kangwon National University, Samcheok, Korea

Objective To examine the characteristics and changes in the pharyngeal phase of swallowing according to fluid viscosity in normal healthy persons, to help determine fluid intake methods in more detail than the use of standardized fluid.

Methods This was a prospective observational study involving 10 normal healthy adults. While the participants sequentially took in fluids with 10 different viscosities changes in the pharyngeal phase of the swallowing process were monitored using videofluoroscopic swallowing studies (VFSS). Twenty parameters of the pharyngeal phase, including epiglottis contact, laryngeal elevation, pharyngeal constriction, and upper esophageal sphincter opening, were determined and compared.

Results No significant viscosity-based changes in epiglottis contact, laryngeal elevation, or upper esophageal sphincter-opening duration of the pharyngeal phase were observed. However, pharyngeal transit time and time from the start of the pharyngeal phase to peak pharyngeal constriction were significantly delayed upon intake of fluid with viscosities of 150.0 centipoise (cP) and $200.0 \mathrm{cP}$.

Conclusion VFSS analysis of fluid intake may require the use of fluids of various concentrations to determine a suitable viscosity of thickener mixture for each subject.

Keywords Dysphagia, Viscosity, Thickener

Received July 21, 2015; Accepted September 11, 2015

Corresponding author: Sun Hong Song

Department of Rehabilitation Medicine, Gangneung Asan Hospital, University of Ulsan College of Medicine, 38 Bangdong-gil, Gangneung 25440, Korea

Tel: +82-33-610-3361, Fax: +82-33-610-4960, E-mail: song0805@gnah.co.kr

(c) This is an open-access article distributed under the terms of the Creative Commons Attribution Non-Commercial License (http://creativecommons. org/licenses/by-nc/4.0) which permits unrestricted noncommercial use, distribution, and reproduction in any medium, provided the original work is properly cited.

Copyright $\odot 2016$ by Korean Academy of Rehabilitation Medicine

\section{INTRODUCTION}

Dysphagia is common in the presence of central nervous system anomalies. It is observed in over $50 \%$ of cases of cerebrovascular accident (CVA), $44 \%$ of multiple sclerosis cases, $60 \%$ of patients with amyotrophic lateral sclerosis, $50 \%$ of traumatic brain injury patients, and in $84 \%$ of patients with other neurodegenerative diseases $[1,2]$. In particular, dysphagia following a CVA is often encountered in rehabilitation medicine, where it presents great difficulties in maintaining quality of life and sup- 
plying nutrition, with an increased risk of serious clinical problems such as asphyxia, aspiration pneumonia, and malnutrition [3]. Aspiration of food or saliva due to dysphagia can lead to respiratory infections, i.e., pneumonia. According to one study, the prevalence of pneumonia in patients with dysphagia was $19 \%$, compared to $8 \%$ in those without dysphagia [4].

Videofluoroscopic swallowing study (VFSS) is generally accepted as the gold standard technique in the diagnosis and treatment of dysphagia [5]. VFSS facilitates comprehensive evaluation of the active features of the oral cavity, pharynx, and esophagus.

Despite the fact that fluid intake test is critical for determining the possibility of a fluid diet and water intake, such tests frequently involve only a few viscosities and do not take into consideration the safest and optimal viscosity. This leads to inconsistencies between fluid intake results by VFSS test and the patient's actual fluid intake pattern, which can lead to aspiration pneumonia. A related study reported that during an ultrathin fluid test in patients in whom intake of thin fluids was safe, aspiration occurred in $50 \%$ of the cases [6]. Although it might be more appropriate to use ultrathin fluid to determine the safety of water intake, accurate observations by fluoroscopy are difficult, and accurate measurement of viscosity in the stepwise treatment process is problematic. Thickeners are used clinically when aspiration of thin fluid, but not thick fluid, is observed. However, the fact that thickener viscosity is generally not measured accurately can lead to difficulties at the bedside. Aspiration can occur if the thickener has inadequate viscosity, which unnecessarily delays treatment of dysphagia. In addition, according to the test diet standards of the American Dietetic Association, because the difference in viscosity between thin and thick fluid is excessive, the correlation between the actual test results and their clinical application is insufficient [7]. The first aim of our study was to determine the safest fluid viscosity for optimal rehabilitation for dysphagic patients who have difficulty swallowing fluids.

Use of thickeners with more appropriate viscosity, based on intake tests using fluids of different viscosities, would improve the treatment of dysphagic patients by allowing safer fluid intake and reducing the risk of aspiration pneumonia. Therefore, the present study aimed to determine a method of the selection of thickeners to optimize the viscosity of dietary fluid intake for dysphagic patients by assessing using the VFSS test the characteristics and pattern of changes in the swallowing process of normal healthy adults when drinking fluids with various viscosities.

\section{MATERIALS AND METHODS}

\section{Participants}

This was a prospective observational preliminary study. The participants consisted of 10 healthy adults ( 8 males and 2 females; mean age of $37.2 \pm 5.1$ years). The study was conducted after receiving the approval of the Institutional Review Board. None of the participants had any swallowing, neurological, or gastrointestinal disorders. To ensure the accuracy of the examination, food intake was prohibited for 4 hours and liquid intake, including water, for 2 hours prior to the examination.

\section{Procedures}

All procedures were performed using a properly collimated radiographic-fluoroscopic unit KF-7 (Shimadzu Corporation, Kyoto, Japan) providing 63 kV, 1.2 mA-type output for the full field-of-view mode (12 inch input phosphor diameter). Studies were conducted in the fluoroscopic contrast study room of our radiology department, with a licensed radio-technician and occupational therapist performing the examination in a space shielded by lead under the supervision of a doctor of rehabilitation medicine. The VFSS images were recorded as highdefinition digital files, using a Samsung HMX-H405 camcorder operating at 30 frames per second. The recorded images were replayed from a PC and analyzed by the authors by taking measurements at $1 / 100$ second on the Adobe Premiere 6.5 multimedia player.

The examinations were conducted with the participants seated sideways on a chair attached to the fluoroscopy unit. The head position was set to neutral, facing forward, and metallic items were removed to prevent artifacts. A lead apron was worn to protect the reproductive organs. The boundaries of the fluoroscopic field of the lateral posture were set as lips anteriorly, nasopharynx superiorly, cervical spine posteriorly, and cervical esophagus inferiorly (Fig. 1).

To reduce errors in the VFSS examination, the method used involved intake of liquid with a specific viscosity, followed by examination with a different concentration 3 minutes later, with sequential intake of liquids of 10 con- 


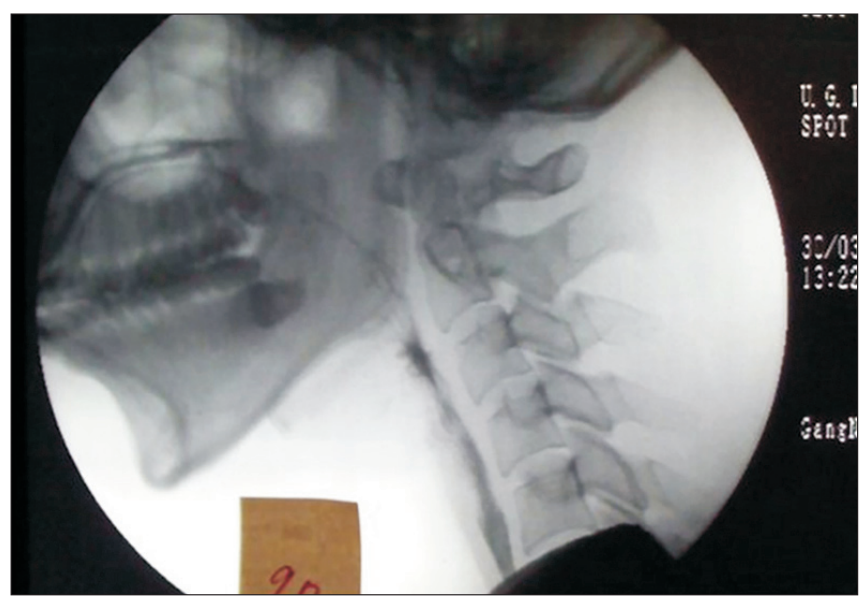

Fig. 1. Boundaries of the lateral fluoroscopic view.

centrations. The viscosity of $\mathrm{BaSO}_{4}$ used in the examination was set to $5.0,10.0,20.0,30.0,40.0,50.0,75.0,100.0$, 150.0 , and 200.0 centipoise (cP), to ensure inclusion of the viscosity of the commercialized fluid widely used in VFSS examinations. A $10 \mathrm{~mL}$ volume of fluid of each viscosity was administered.

The viscosities of the test materials were checked and the concentration curves assessed using a viscometer DV-II+Pro (Brookfield Engineering Laboratories, Middleboro, MA, USA) (Fig. 2).

\section{Measures}

Previous researchers used different definitions of the pharyngeal phase of swallowing [8,9]. In the present study, we used the posterior nasal spine as a landmark for the beginning of the pharyngeal phase, and the moment when the upper esophageal sphincter (UES) closes and the bolus has fully entered the esophagus as the end of the pharyngeal phase [8]. Thus, the pharyngeal transit time (PTT) was defined as the interval from the beginning of the pharyngeal phase to closure of the UES. The epiglottis contact was defined as the arrival of the bolus head in the valleculae. The latency of laryngeal elevation (LLE) was defined as the interval from the beginning of the pharyngeal phase to laryngeal elevation. The peak laryngeal elevation was defined as the maximal point of anterior and superior excursion of the larynx during swallowing [8]. The rise time of laryngeal elevation was defined as the interval from the latency of laryngeal elevation to the latency of peak laryngeal elevation. The duration of laryngeal elevation was defined as the interval

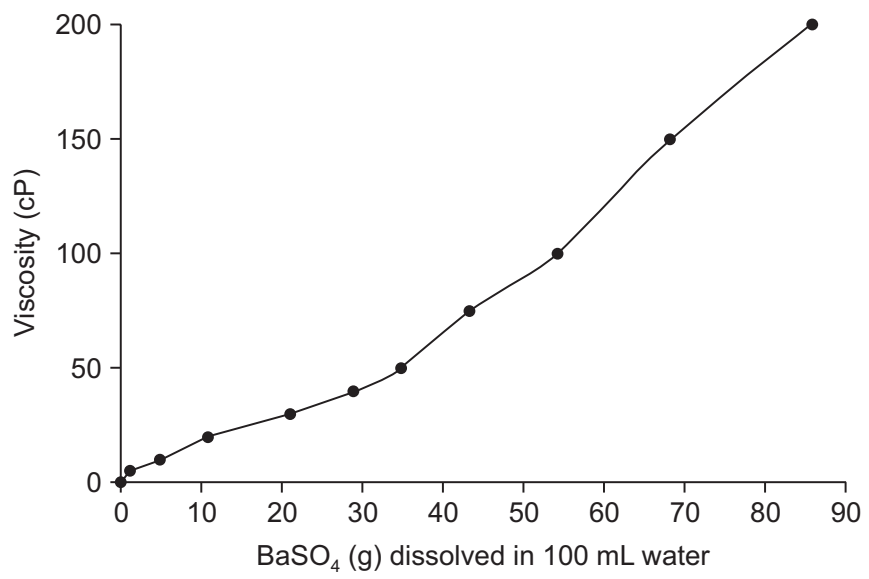

Fig. 2. The curve of viscosity and $\mathrm{BaSO}_{4}$ dissolved in water $(100 \mathrm{~mL})$. The temperature was $21.8^{\circ} \mathrm{C} \pm 2.1^{\circ} \mathrm{C}$.

between the initiation and the end of laryngeal elevation. As the bolus is propelled into the upper esophagus, the pharynx is typically completely obliterated by the tongue, which pushes against the contracting posterior pharyngeal wall. Pharyngeal constriction was defined as the beginning of pharyngeal wall and soft palate constriction. Peak pharyngeal constriction was defined as the moment when the anterior-posterior diameter of the pharyngeal wall becomes narrowest [9]. The rise time of pharyngeal constriction was defined as the interval from the latency of pharyngeal constriction to the latency of peak pharyngeal constriction. The UES opening was identified as the moment when the narrowest part of the upper esophagus (between C4 and C6) opened; this opening is the most functionally significant $[8,9]$.

We measured the values of 20 distinct timing variables during the pharyngeal phase of swallowing. Table 1 shows the abbreviations and definitions used [8].

\section{Statistical analysis}

The SPSS ver. 21.0 software (IBM, Armonk, NY, USA) was employed for statistical analyses. Firstly, differences in 20 parameters among the viscosities were analyzed by ANOVA. If any parameter had significant differences across bolus viscosity, the Bonferroni method was used for post hoc analysis. Values were presented as means \pm standard deviation. A significance level of $\alpha=0.05$ was determined. 
Table 1. Abbreviations and definitions

\begin{tabular}{ll}
\multicolumn{1}{c}{ Variable } & \\
\hline LEC & Interval between the initiation of pharyngeal phase and epiglottis contact \\
LLE & Interval between the initiation of pharyngeal phase and the initiation of laryngeal elevation \\
LPC & Interval between the initiation of pharyngeal phase and the initiation of pharyngeal constriction \\
LUEO & Interval between the initiation of pharyngeal phase and the initiation of upper esophageal sphincter opening \\
LPLE & Interval between the initiation of pharyngeal phase and the peak laryngeal elevation \\
LPPC & Interval between the initiation of pharyngeal phase and the peak pharyngeal constriction \\
PTT & Interval between the initiation of pharyngeal phase and closure of pharyngoesophageal sphincter \\
LLE-LEC & Interval between latency of epiglottis contact and latency of laryngeal elevation \\
LPC-LLE & Interval between latency of laryngeal elevation and latency of pharyngeal constriction \\
LPPC-LLE & Interval between latency of laryngeal elevation and latency of peak pharyngeal constriction \\
LUEO-LLE & Interval between latency of laryngeal elevation and latency of upper esophageal sphincter opening \\
LPLE-LPC & Interval between latency of peak pharyngeal constriction and latency of peak laryngeal elevation \\
LPPC-LPLE & Interval between latency of peak laryngeal elevation and latency of peak pharyngeal constriction \\
LPLE-LUEO & Interval between latency of upper esophageal sphincter opening and latency of peak pharyngeal constriction \\
LPLE-LLE & Rise time of laryngeal elevation \\
DLE & Interval between the initiation and the end of laryngeal elevation \\
LPPC-LPC & Rise time of pharyngeal constriction \\
DUEO & Interval between the opening and closing of upper esophageal sphincter \\
LUEO-LPC & Interval between latency of pharyngeal constriction and latency of upper esophageal sphincter opening \\
LUEO-LPPC & Interval between latency of peak laryngeal constriction and latency of upper esophageal sphincter opening \\
\hline
\end{tabular}

\section{RESULTS}

The interval between the initiation of the pharyngeal phase and epiglottis contact (LEC); laryngeal elevation (LLE); pharyngeal constriction (LPC); and upper esophageal sphincter opening (LUEO) did not differ significantly according to viscosity. The interval between the initiation of the pharyngeal phase and the peak laryngeal elevation (LPLE) also did not show a statistically significant difference according to viscosity ( $\mathrm{p}>0.05)$.

However, the interval between the initiation of the pharyngeal phase and the peak pharyngeal constriction differed significantly according to viscosity. In particular, delays were observed at viscosities of 150.0 and $200.0 \mathrm{cP}(\mathrm{p}<0.05)$.

Furthermore, the PTT differed significantly according to viscosity. In particular, statistically significant delays were observed at viscosities of 150.0 and $200.0 \mathrm{cP}(\mathrm{p}<0.01)$.

The gaps between LLE-LEC, LPC-LLE, LPPC-LLE, LPLE-LPC, LPPC-LPLE, LPLE-LUEO, and LPLE-LLE, which express the intervals of each movement, did not differ significantly according to viscosity $(\mathrm{p}>0.05)$.

The interval between the initiation and the end of laryngeal elevation (DLE) and the interval between the opening and closing of the upper esophageal sphincter (DUEO) also did not differ significantly according to viscosity ( $p>0.05)$. The intervals of LPPC-LPC, LUEO-LPC, and LUEO-LPPC, which express the gaps between each movement, also did not differ significantly according to viscosity ( $\mathrm{p}>0.05)$.

The results described above are shown in Table 2, Figs. 3 and 4.

\section{DISCUSSION}

This study was conducted to provide guidelines on fluid intake by determining the optimal viscosity of thickener to use in clinical settings based on the kinematic changes in the pharyngeal phase observed on VFSS when normal healthy adults took in fluids with various viscosities. In 2002, the American Dietetic Association established the National Dysphagia Diet (NDD) guidelines for thickened dietary supplements [7]. According to these guidelines, thin fluid should be 1-50 cP, Neckar-like fluid 51-350 cP, honey-like fluid 351-1,750 cP, and thick fluid >1,750 cP. The appropriate viscosity of fluid for dysphagic patients for intake or testing is determined on this basis. However, 


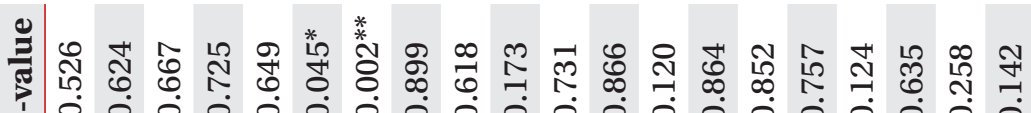

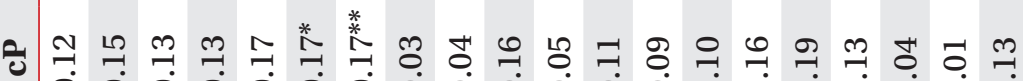

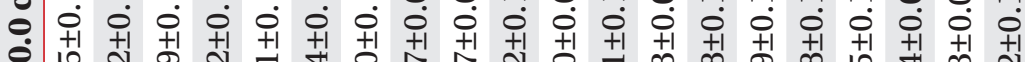

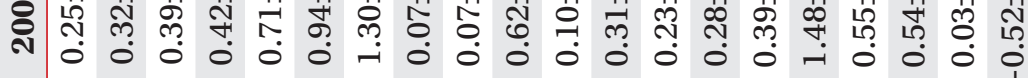

근 근 는

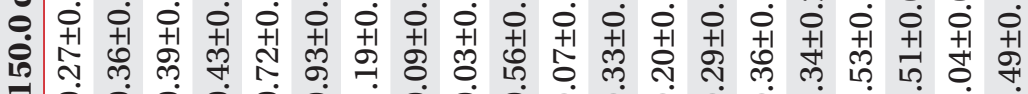

に2

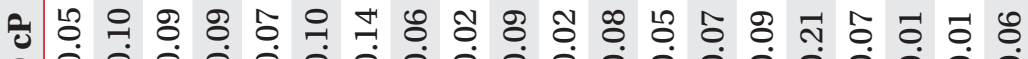

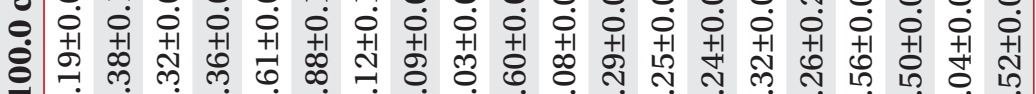

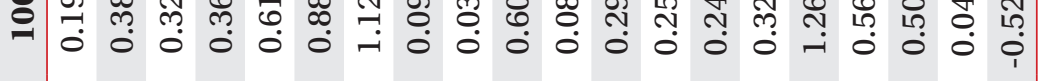

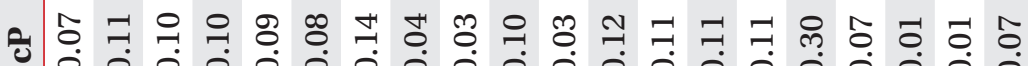

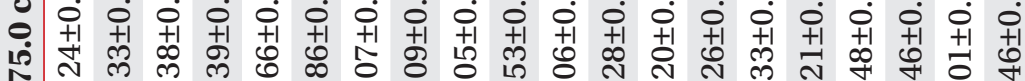

N̦

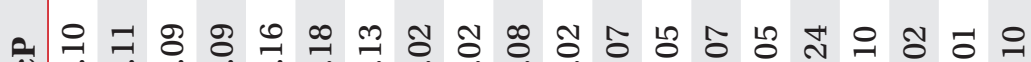

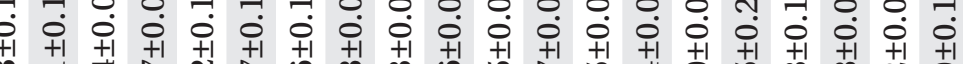

.

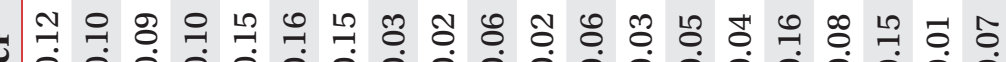

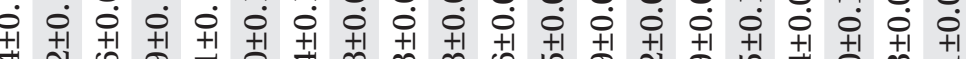

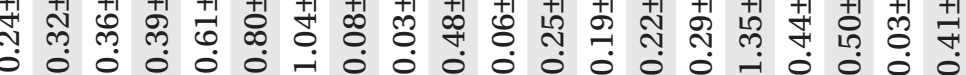

๑ ๕ ชิ

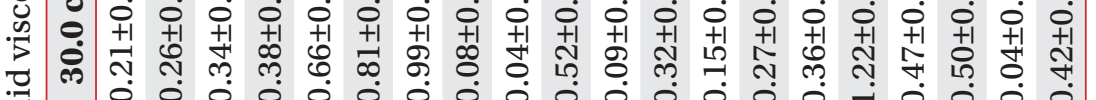

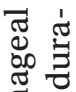

ㄷํㄱำ

के

过

골

जै

읍

ฮี

호

琴

芒旨

.己.

范

욜

ฮิ

정

골

प듕

気 ฮี

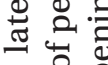

0 응

焉

氞

ज放

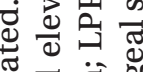

ส

믈

름

๑ \& $\exists$ व

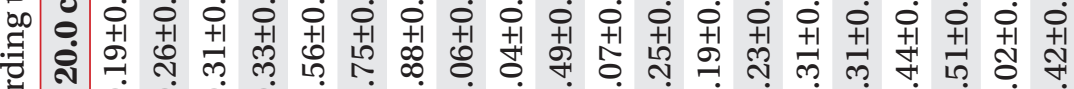

층

뉴

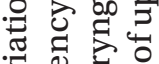

ज

๑ 过

면

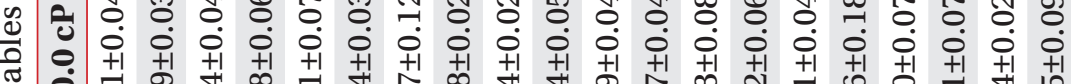

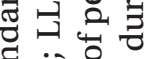

范

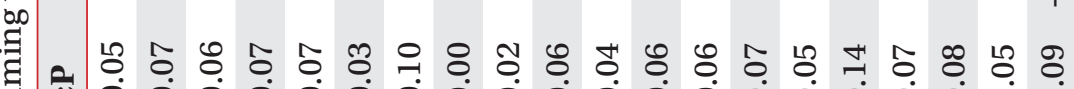

跑

đิ

I

है ज्ञ

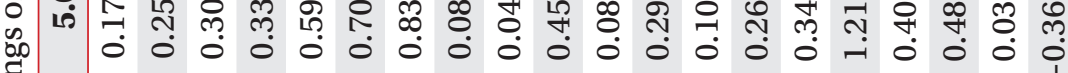

을

递

屯ี

全

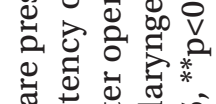
क जै 

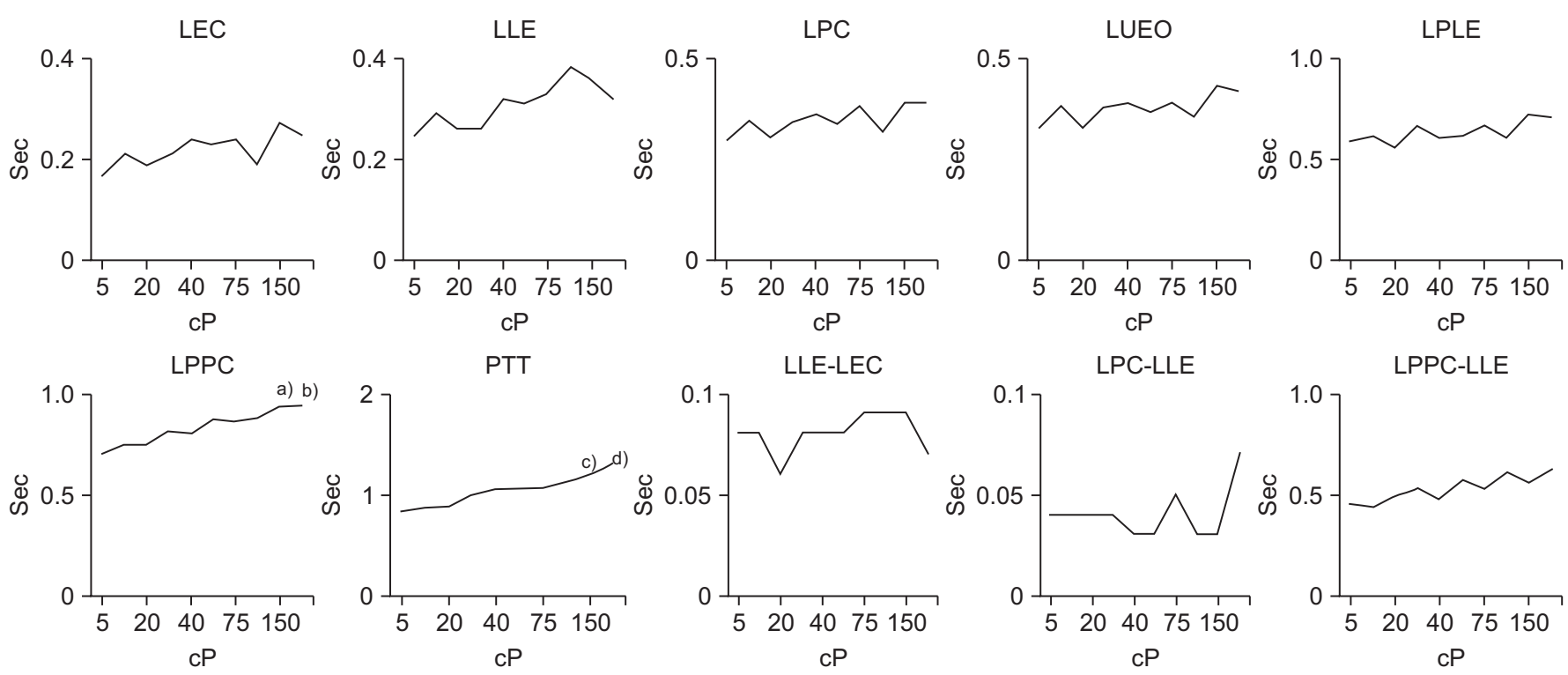

Fig. 3. Findings of timing variables according to liquid viscosity in normal subjects. LEC, latency of epiglottis contact; LLE, latency of laryngeal elevation; LPC, latency of pharyngeal constriction; LUEO, latency of upper esophageal sphincter opening; LPLE, latency of peak laryngeal elevation; LPPC, latency of peak pharyngeal constriction; PTT, pharyngeal transit time. ${ }^{\text {a) }} \mathrm{p}<0.05$ at $150 \mathrm{cP}$ on LPPC, ${ }^{\text {b) }} \mathrm{p}<0.05$ at $200 \mathrm{cP}$ on LPPC, ${ }^{\mathrm{c}} \mathrm{p}<0.01$ at $150 \mathrm{cP}$ on PPT, ${ }^{\text {d) }} \mathrm{p}<0.01$ at $200 \mathrm{cP}$ on PPT.
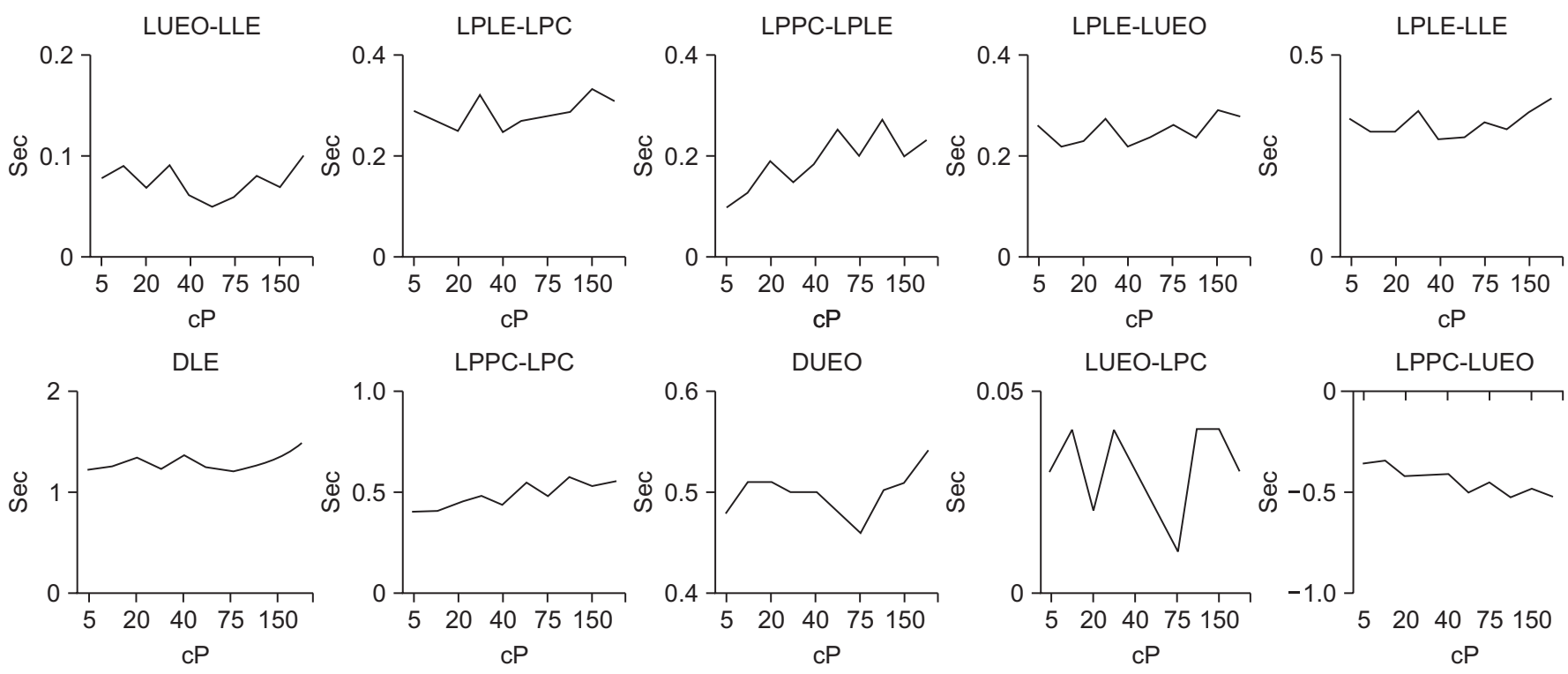

Fig. 4. Findings of timing difference variables according to liquid viscosity in normal subjects. LEC, latency of epiglottis contact; LLE, latency of laryngeal elevation; LPC, latency of pharyngeal constriction; LUEO, latency of upper esophageal sphincter opening; LPLE, latency of peak laryngeal elevation; LPPC, latency of peak pharyngeal constriction; PTT, pharyngeal transit time; DLE, duration of laryngeal elevation; DUEO, duration of upper esophageal sphincter opening.

the fluid used in tests conducted at most hospitals use a commercialized viscosity, and phased diets for dysphagia severity are prescribed according to the guidelines of the nutritional unit of each hospital. Therefore, regarding the possibility of fluid intake associated with hydration, conducting tests with only a few commercialized fluids 
makes determining the safest fluid viscosity for dysphagic patients problematic. When testing with thick fluid shows laryngeal penetration without definite aspiration, and testing with thin fluid shows definite aspiration, this creates a dilemma on how to determine the appropriate fluid viscosity for that dysphagic patient. Moreover, a rough visual determination of the viscosity of a thickened mixture can be required, which can in extreme cases lead to development of aspiration pneumonia.

Most rehabilitation units use commercialized thickener powder to prevent dehydration in dysphagic patients by enabling them to drink a sufficient quantity of water. This thickener is used mainly when mild penetration without definite laryngeal aspiration with thick fluid, and definite aspiration with thin fluid, is seen on VFSS. However, because the exact viscosity is not set, the mixture can easily be made too thin, increasing the risk of aspiration pneumonia.

Increasing the bolus viscosity reduces the laryngeal penetration of the bolus, which decreases the risk of aspiration and significantly improves the stability and efficiency of deglutition [10]. Moreover, increasing the viscosity has been reported to reduce the risks of laryngeal penetration or aspiration by extending the oral and pharyngeal phase transit time, delaying pharyngoesophageal sphincter (PES) opening, and increasing the duration of pharyngeal peristaltic waves [11]. However, it also increases the risk of post-swallowing aspiration by increasing the amount of post-swallow residue in the pharynx, which leads to the use of compensatory mechanisms such as the chin tuck [11]. In addition, observations of the pharyngeal phase of the process by computed tomography using thin and thick fluid showed a greater difference in true vocal cord closure with thin than thick fluid, while there were no significant changes in epiglottis movement, hyoid movement, and PES opening duration [12]. This suggests that the pharyngeal transit time is reduced with thin fluid, which leads to more rapid true vocal cord closure to reduce the aspiration risk. However, the limited range of viscosities used in the above studies, prevents determination of the viscosity that induces significant changes in parameters. As such, this study used fluids of a wide range of viscosities.

According to our results, intake of a thin-fluid mixture that contained barium (viscosity, $50 \mathrm{cP}$ ) and thickfluid mixture that contained barium (viscosity, $250 \mathrm{cP}$ ), resulted in an increase in PTT and delayed peak pha- ryngeal constriction at viscosities of $150 \mathrm{cP}$ and $\geq 200 \mathrm{cP}$, respectively. Moreover, parameters such as epiglottis movement and laryngeal elevation were not affected by fluid viscosity. These results are consistent with previous reports. However, there was a significant difference with intake of fluid of 150-200 cP, which would be considered a mid-thick viscosity fluid [9]. Therefore, tests using fluid of mid-thick viscosity would enable a strategy for increasing fluid intake to be established.

When fluid is taken in at the bedside, a commercial thickener is commonly used. If aspiration is observed with thin but not thick fluid, we suggest that fluid of viscosity 150-200 cP be used, which would enable safer fluid intake training. When fluid is taken in, significant differences in PTT and the time to reach peak pharyngeal constriction are observed at viscosities $\geq 150 \mathrm{cP}$, suggesting that this can also be applied to the thickener.

The limitations of this study were as follows. First, bolus volume was not varied. According to Clave et al. [10], bolus volume significantly affects the stability and efficiency of deglutition. However, a set volume of $10 \mathrm{~mL}$ was used in this study. Therefore, a range of bolus volumes should be evaluated in future studies. Second, the appropriate viscosity was not verified in actual dysphagic patients. This warrants follow-up studies in patients capable of fluid intake to identify the optimal viscosity for individual dysphagic patients.

According to Lazarus et al. [13], normal persons showed a longer cricopharyngeal opening on paste boluses, while dysphagic patients did not show these changes. This may be because the motor-sensory pathways controlling these systematic swallow variations have been compromised in these patients. Therefore, our results may not be applicable to all patients with dysphagia. Future studies should focus on validating our results in patients with actual swallowing difficulties, and this is planned as a future research project. In addition, when PTT is delayed, the risk of aspiration increases. Patients with dysphagia are likely more vulnerable than normal persons because of their altered motor-sensory pathway.

Third, poor fluoroscopic imaging quality was seen with very-low-viscosity fluid, which made the images indecipherable. This is a technical issue that should be overcome in future.

In conclusion, we observed significant delays in PTT and time to reach peak pharyngeal constriction with mid- 
thick fluid; i.e., fluid of viscosity intermediate between thin and thick fluid, on VFSS conducted. Therefore, it is recommended that in future various concentrations of fluid should be used in VFSS tests to enhance safety and optimize fluid intake for dysphagic patients. In addition, if a thickener is required to prevent aspiration, the safe concentration of test fluid and thickener must be determined to ensure safe and optimal use of thickener.

This technique will enable regulation of the amount of thickener used in dysphagic patients. Further studies using various bolus volumes and viscosities are required in patients with dysphagia.

\section{CONFLICT OF INTEREST}

No potential conflict of interest relevant to this article was reported.

\section{REFERENCES}

1. Cook IJ, Kahrilas PJ. AGA technical review on management of oropharyngeal dysphagia. Gastroenterology 1999;116:455-78.

2. Clave P, Terre R, de Kraa M, Serra M. Approaching oropharyngeal dysphagia. Rev Esp Enferm Dig 2004; 96:119-31.

3. Gordon C, Hewer RL, Wade DT. Dysphagia in acute stroke. Br Med J (Clin Res Ed) 1987;295:411-4.

4. Smithard DG, O'Neill PA, Parks C, Morris J. Complications and outcome after acute stroke. Does dysphagia matter? Stroke 1996;27:1200-4.

5. Martin-Harris B, Jones B. The videofluorographic swallowing study. Phys Med Rehabil Clin N Am 2008; 19:769-85.

6. Fink TA, Ross JB. Are we testing a true thin liquid? Dysphagia 2009;24:285-9.

7. Strowd L, Kyzima J, Pillsbury D, Valley T, Rubin B. Dysphagia dietary guidelines and the rheology of nutritional feeds and barium test feeds. Chest 2008;133: 1397-401.

8. Kendall KA, McKenzie S, Leonard RJ, Goncalves MI, Walker A. Timing of events in normal swallowing: a videofluoroscopic study. Dysphagia 2000;15:74-83.

9. Lee SI, Yoo JY, Kim M, Ryu JS. Changes of timing variables in swallowing of boluses with different viscosities in patients with dysphagia. Arch Phys Med Rehabil 2013;94:120-6.

10. Clave P, de Kraa M, Arreola V, Girvent M, Farre R, Palomera E, et al. The effect of bolus viscosity on swallowing function in neurogenic dysphagia. Aliment Pharmacol Ther 2006;24:1385-94.

11. Dantas RO, Kern MK, Massey BT, Dodds WJ, Kahrilas PJ, Brasseur JG, et al. Effect of swallowed bolus variables on oral and pharyngeal phases of swallowing. Am J Physiol 1990;258(5 Pt 1):G675-81.

12. Inamoto Y, Saitoh E, Okada S, Kagaya H, Shibata S, Ota $\mathrm{K}$, et al. The effect of bolus viscosity on laryngeal closure in swallowing: kinematic analysis using 320row area detector CT. Dysphagia 2013;28:33-42.

13. Lazarus CL, Logemann JA, Rademaker AW, Kahrilas PJ, Pajak T, Lazar R, et al. Effects of bolus volume, viscosity, and repeated swallows in nonstroke subjects and stroke patients. Arch Phys Med Rehabil 1993;74: 1066-70. 